\title{
Editorial: Friction and Multi-Field Problems in Sliding Contacts
}

\author{
Rostyslav Martynyak ${ }^{1}$, Elena Torskaya ${ }^{2}$ and Yang $\mathrm{Xu}^{3 *}$ \\ ${ }^{1}$ Pidstryhach Institute for Applied Problems of Mechanics and Mathematics (NAN Ukraine), Lviv, Ukraine, ${ }^{2}$ Institute for \\ Problems in Mechanics (RAS), Moscow, Russia, ${ }^{3}$ School of Engineering, University of Glasgow, Glasgow, United Kingdom
}

Keywords: contact mechanics and tribology, friction, wear, frictional heat generation, multi-field problems

\section{Editorial on the Research Topic}

\section{Friction and Multi-Field Problems in Sliding Contacts}

Friction inevitably occurs in the form of shear stress between rubbing surfaces in machine components and many natural structures because of the solid interaction and adhesion. In the early days of tribology, few classic frictional problems (e.g., Mindlin, 1949) associated with simple contact geometries can be solved analytically using the theory of elasticity and linear viscoelasticity (Johnson, 1987; Goryacheva, 1998). Thanks to the fast growth of computational power, friction problems with complex interfaces, complicated material models and damage mechanism are solved numerically using finite element method (Wriggers, 2002). Besides the complex mechanical field, the performance and service life of the contact interface is largely influenced by multi-fields, e.g., thermal, electric, magnetic, acoustic and, chemical field (Wang and Zhu, 2019). Thermal field is commonly coupled with the mechanical field due to the heat generation between the contacting asperities, especially at high sliding speed. One of the representative phenomena thermoelastic instability (Barber, 2018). Thermal stresses can induce partial slip of surfaces in the vicinity of thermoinsulated (Malanchuk et al., 2011) and medium-filled (Chumak et al., 2014) surface irregularities. Electrical connector is a typical example of coupled mechanical-thermal-electrical problem (Holm, 2013). The asperity contact between mating terminals greatly constricts the electric field so that the current only flows through the tiny contact area which causes a larger contact resistance and higher energy waste through Joule heating. In the finger-pad interaction, the tribo-charges can enhance the adhesion between skin and pad through the Coulomb force. This can eventually alter the real contact area and friction (Persson, 2018). Triboelectric nanogenerator (TENG) is another heat area where the mechanical field is strongly coupled with the electromagnetic field (Wang et al., 2016). TENG relies on the tribo-charges, generated within the real contact area between two sliding surfaces, to transform the kinetic energy into the electricity (Xu et al., 2020). Magnetic field can be either measured by using TENG as a magnetic sensor (Yang et al., 2012) or can be used to achieve the wireless power transmission (Cao et al., 2019). The acoustic method is commonly as an alternative to reduce the friction in machine components (Mahajan et al., 2008). In other areas, e.g., haptics (Wiertlewski et al., 2016) and ultrasonic actuator (Zhao, 2011), it is applied to tune the friction. The chemical field is commonly coupled with thermal field and other environmental factors. Heat generation between contacting asperities accelerate the chemical reaction on the interface. Important topics include the formation of tribo-film (Spikes, 2004; Brizmer et al., 2017) and tribo-emission (Wang et al., 2019).

A total of six papers are included in this article collection. At the single asperity contact level, the classic Cattaneo-Mindlin partial slip problem was extended to cover more complex interfacial geometry (Klimchuk and Ostryk). The bilinear strain hardening law was introduced to the seminar work of Jackson and Green asperity contact model (Ghaednia et al.). Effect of surface layer on the sliding contact between a rigid indenter and a viscoelastic half-space was studied using 
boundary element method (Torskaya and Stepanov). At the rough surface contact level, the thermal rectification between sinusoidal waviness surfaces with trapped interstitial gas was solved using an analyticonumerical approach (Chumak and Martynyak). A simplified theory of electroadhesion for rough interface was built upon the Persson's theory of contact (Ciavarella and Papangelo). A simple estimation of the adhesion forces between rough surfaces was achieved using bearing area model. Finally, an analytical framework for developing multiscale thermo-electro-mechanical approach for rough surface contact was proposed (Komvopoulos).

\section{REFERENCES}

Barber, J. R. (2018). Contact Mechanics. Springer. doi: 10.1007/978-3-319-70939-0 Brizmer, V., Matta, C., Nedelcu, I., and Morales-Espejel, G. E. (2017). The influence of tribolayer formation on tribological performance of rolling/sliding contacts. Tribol. Lett. 65:57. doi: 10.1007/s11249-017-0839-3

Cao, S., Zhang, H., Guo, R., Zhang, W., and Sang, S. (2019). Wireless power transmission enabled by a triboelectric nanogenerator via a magnetic interaction. Energy Technol. 7:1900503. doi: 10.1002/ente.2019 00503

Chumak, K., Malanchuk, N., and Martynyak, R. (2014). Partial slip contact problem for solids with regular surface texture assuming thermal insulation or thermal permeability of interface gaps. Int. J. Mech. Sci. 84, 138-146. doi: 10.1016/j.ijmecsci.2014.04.015

Goryacheva, I. G. (1998). Contact Mechanics in Tribology. Dordrecht: Kluwer Academic Publishers. doi: 10.1007/978-94-015-9048-8

Holm, R. (2013). Electric Contacts: Theory and Application. Springer.

Johnson, K. L. (1987). Contact Mechanics. Cambridge: Cambridge University Press.

Mahajan, M., Jackson, R., and Flowers, G. (2008). Experimental and analytical investigation of a dynamic gas squeeze film bearing including asperity contact effects. Tribol. Trans. 51, 57-67. doi: 10.1080/104020007017 39339

Malanchuk, N., Martynyak, R., and Monastyrskyy, B. (2011). Thermally induced local slip of contacting solids in vicinity of surface groove. Int. J. Solids Struct. 48, 1791-1797. doi: 10.1016/j.ijsolstr.2011. 02.028

Mindlin, R. D. (1949). Compliance of elastic bodies in contact. ASME J. Appl. Mech. $16,259-268$.

Persson, B. N. J. (2018). The dependency of adhesion and friction on electrostatic attraction. J. Chem. Phys. 148:144701. doi: 10.1063/1.5024038

Spikes, H. (2004). The history and mechanisms of ZDDP. Tribol. Lett. 17, 469-489. doi: 10.1023/B:TRIL.0000044495.26882.b5
We hope the readers can find the latest accomplishment in the multi-field modeling of the tribological problems. We would like to thank all authors contributing in this article collection.

\section{AUTHOR CONTRIBUTIONS}

YX: Writing-original draft. RM and ET: Writing-review and editing. All authors contributed to the article and approved the submitted version.

Wang, J., and Zhu, D. (2019). Interfacial Mechanics: Theories and Methods for Contact and Lubrication. Boca Raton, FL: CRC Press. doi: 10.1201/9780429131011

Wang, Y., Yamada, N., Xu, J., Zhang, J., Chen, Q., Ootani, Y., et al. (2019). Triboemission of hydrocarbon molecules from diamond-like carbon friction interface induces atomic-scale wear. Sci. Adv. 5:eaax9301. doi: 10.1126/sciadv.aax9301

Wang, Z. L., Lin, L., Chen, J., Niu, S., and Zi, Y. (2016). Triboelectric Nanogenerators. Springer. doi: 10.1007/978-3-319-40039-6

Wiertlewski, M., Friesen, R. F., and Colgate, J. E. (2016). Partial squeeze film levitation modulates fingertip friction. Proc. Natl. Acad. Sci. U.S.A. 113, 9210-9215. doi: 10.1073/pnas.1603908113

Wriggers, P. (2002). Computational Contact Mechanics. New York, NY: Wiley.

Xu, Y., Min, G., Gadegaard, N., Dahiya, R., and Mulvihill, D. M. (2020). A unified contact force-dependent model for triboelectric nanogenerators accounting for surface roughness. Nano Energy 76:105067. doi: 10.1016/j.nanoen.2020.105067

Yang, Y., Lin, L., Zhang, Y., Jing, Q., Hou, T. C., and Wang, Z. L. (2012). Selfpowered magnetic sensor based on a triboelectric nanogenerator. ACS Nano 6, 10378-10383. doi: 10.1021/nn304374m

Zhao, C. (2011). Ultrasonic Motors. Berlin: Springer. doi: $10.1007 / 978-3-642-15305-1$

Conflict of Interest: The authors declare that the research was conducted in the absence of any commercial or financial relationships that could be construed as a potential conflict of interest.

Copyright (c) 2020 Martynyak, Torskaya and Xu. This is an open-access article distributed under the terms of the Creative Commons Attribution License (CC BY). The use, distribution or reproduction in other forums is permitted, provided the original author(s) and the copyright owner(s) are credited and that the original publication in this journal is cited, in accordance with accepted academic practice. No use, distribution or reproduction is permitted which does not comply with these terms. 